\section{Critically Evaluated Propagation Rate Coefficients in Free-Radical Polymerizations: Part III. Methacry- lates with Cyclic Ester Groups (IUPAC Technical Report)}

\section{S. Beuermann \\ Pure and Applied Chemistry \\ Vol. 75, No. 8, pp. 1091-1096 (2003)}

Modeling and optimization of free-radical polymerization processes requires the knowledge of accurate individual rate coefficients for the reactions occurring during a polymerization. Pulsed laser-initiated polymerization in conjunction with polymer analysis by sizeexclusion chromatography has proven to be a valuable technique to derive propagation rate coefficients $k_{\mathrm{p}}$. The IUPAC Working Party on Modeling of Polymerization Kinetics and Processes has reported critically evaluated rate coefficient data for styrene and alkyl methacrylates. This paper continues the series by reporting propagation rate coefficients, $k_{p}$, as a function of temperature, for bulk free-radical homopolymerizations of oxiranylmethyl, cyclohexyl, and benzyl methacrylate at ambient pressure and low conversion. For each monomer, the rate coefficients have been independently measured in two laboratories. The data determined from experiments carried out in independent laboratories obey the consistency criteria established for this technique. The rate coefficients for the three monomers are well represented by a single Arrhenius relation.

\section{www.iupac.org/publications/pac/2003/7508/7508x1091.html}

\section{Minimum Requirements for}

Reporting Analytical Data for Environmental Samples (IUPAC Technical Report)

\section{H. Egli, M. Assenakis, H. Garelick, R. Van}

Grieken, W. Peijnenburg, L. Klasinc, W. Kördel,

\section{N. Priest, and T. Tavares}

Pure and Applied Chemistry

Vol. 75, No. 8, pp. 1097-1106 (2003)

Environmental analytical data are generated to investigate how human activities influence the environment to develop, calibrate and validate environmental models, to test whether either standards or quality criteria are exceeded, and to deduce whether there is a potential or actual risk to ecosystems. In addition to the many purposes they serve, published environmental data often attract extra attention from the general press and public, and may even be used to support advisory or regulatory measures. It is, therefore, in the interest of all involved parties to endeavour to publish only data with a proven quality, known uncertainty, and with sufficient additional information about the sample history.

Under the umbrella of the IUPAC Chemistry and the Environment Division, a project team of scientists from different fields of interests have published this paper to provide recommendations for the minimum requirements for reporting environmental-analytical data. Irrespective of the analyte(s) and the goal of the study, the recommendations give general guidance regarding the minimum information that should be provided to adequately describe the sampling strategy, the method of sampling, the sample properties, all handling between sampling and analysis (including storage conditions, pre-treatments, homogenization, sub-sampling), and the analytical methodology (including calculation and validation procedures). The paper provides specific guidance on the environmental compartments: soil, pore water, ground water, inland surface water, sediment, seawater, precipitation water, and air.

Environmental analytical chemists are the intended audience for this guidance paper. If they follow the recommendations, the utility of the published data for the scientific community would be much improved. Full and adequate use of data is only possible if sufficient information is provided. Editors of journals and reviewers of papers submitted for publication are also encouraged to take into consideration the guidance paper. They are key players in the entire publication process and, in taking seriously their responsibility for a high scientific standard of published articles, should consider refusing papers containing environmental-analytical results if the minimum requirements are not met.

\section{www.iupac.org/publications/pac/2003/7508/7508x1097.html}

\section{Atomic Weights of the Elements 2001 (IUPAC Technical Report)}

\section{R. D. Loss}

Pure and Applied Chemistry

Vol. 75, No. 8, pp. 1107-1122 (2003)

The Commission on Atomic Weights and Isotopic 
Abundances wishes to emphasize the need for new precise calibrated isotope composition measurements in order to improve the atomic weights of a number of elements, which are still not known to a satisfactory level of accuracy. However, for many elements the limited accuracy of measurements is overshadowed by terrestrial variability, which is included in the tabulated uncertainty of the atomic weights.

The range of terrestrial variation observed in the atomic weights for most elements is generally small and does not affect most chemists in their day-to-day work. However, as improvements in instrumentation and analyst skill continue to produce more accurate and precise results, analysts may need to seriously consider atomic weight variations, and in some cases actually measure the atomic weights of specific elements in the material they are analyzing. Variations in the atomic weights of elements down to the microscale level also have major benefits, such as the ability to characterize materials not only on their chemical composition but also by their isotopic or atomic weight variability. This variation has been used successfully for many years in fields such as isotope geochemistry and nuclear astrophysics, and is now opening up whole new fields of study in medicine, forensics, and human nutrition. The Commission's task of evaluating atomic weight data has thus expanded

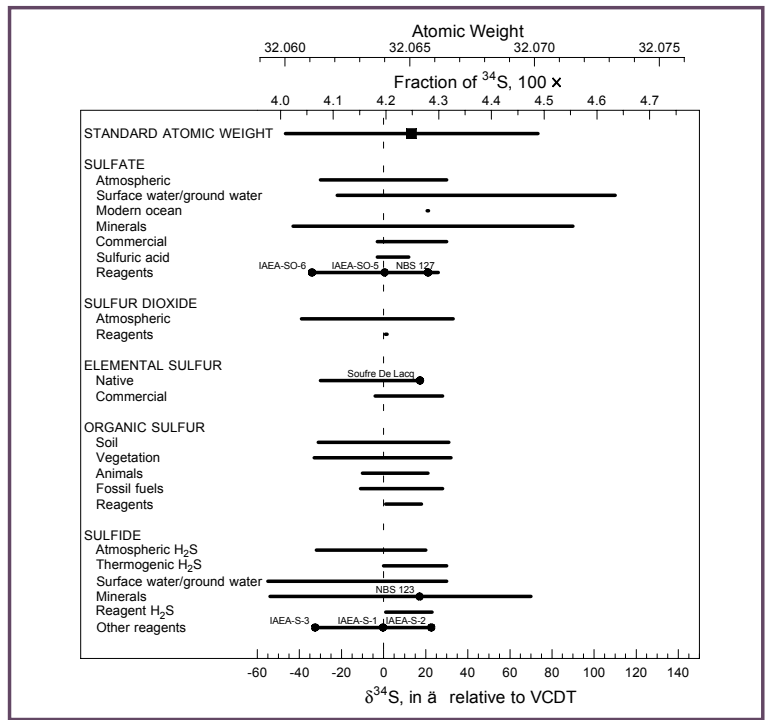

The variation in the atomic weight of sulfur due to natural variation in its isotopic composition (shown here for the fraction of the ${ }^{34} \mathrm{~S}$ isotope). The natural variation in the atomic weight of $S$ places a significant limit on the final uncertainty with which the atomic weight for this element may be stated. [fig reproduced from T.B. Coplen et al., Pure Appl. Chem. 74(10), 1987-2017, 2002] into reviewing isotopic abundance literature in increasingly diverse fields. The Commission is therefore seeking the assistance of all isotopic analysts in reporting isotopic data in a specific and comprehensive manner. This issue is being addressed in a current project [\# 2001-019-2-200] on "Guidelines for mass spectrometric isotope ratio measurement."

\section{www.iupac.org/publications/pac/2003/7508/7508x1107.html}

\section{Regulatory Limits for Pesticide Residues in Water}

\section{J. Hamilton, Á. Ambrus, R. M. Dieterle, A. S.} Felsot, C. A. Harris, P. T. Holland, A. Katayama, N. Kurihara, J. Linders, J. Unsworth, S.-S. Wong Pure and Applied Chemistry

Vol. 75, No. 8, pp. 1123-1155 (2003)

This paper, produced by the IUPAC Commission on Agrochemicals and the Environment, provides guidelines for setting limits on pesticide residues in water. National governments introduced residue limits and guideline levels for pesticide residues in water when policies were implemented to minimize the contamination of ground water and surface water. Initially, governments mainly focused on drinking water.

Contamination of ground water by pesticide residues was for many years generally regarded as unlikely because the soil profile acts as a purifying filter. Residue contamination of surface waters was regarded as transitory because the focus was on the old organochlorine pesticides, which were attached to particulate matter and generally disappeared from clear water. In the early 1980s information had accumulated that some herbicide compounds, which were generally more water-soluble and more widely used than the organochlorines, were being detected in both surface and ground waters. Policies were developed to reduce contamination of ground and surface water and regulatory limits and guideline levels were introduced for residues in drinking water.

Setting regulatory limits for pesticide residues in waters is complex. First we must define the type of water relevant to the proposed limit (e.g., drinking water, reservoir water, lakes and streams, ground water, water for aquaculture, irrigation water, and drinking water for farm animals). Secondly, should we adopt a risk-based approach, a "no more than reasonable if good practices are followed" approach, or a combination of the two? Different approaches will lead 